\title{
Uma análise da dispensa pública do metilfenidato no Brasil: o caso do Espírito Santo
}

\section{| ${ }^{1}$ Luciana Vieira Caliman, ${ }^{2}$ Nathalia Domitrovic |}

Resumo: A expansão do transtorno do déficit de atenção e hiperatividade (TDAH) e o crescimento global do consumo do psicoestimulante metilfenidato, indicado para seu tratamento, são desafios atuais de saúde pública em várias partes do mundo. Esta pesquisa visou investigar a dispensa pública do cloridrato de metilfenidato pelo Sistema Único de Saúde brasileiro (SUS), com ênfase no Estado do Espírito Santo (ES). Realizou-se um mapeamento das políticas de assistência farmacêutica das unidades federativas do país, através de contatos telefônicos e consultas nos seus sites oficiais. Verificou-se que entre as Assistências Farmacêuticas estaduais do Brasil, apenas quatro possuem listas padronizadas de dispensa de medicamentos que incluem o metilfenidato, estando entre elas a do ES. As características e variações da demanda e consumo do metilfenidato, registradas nas Farmácias Cidadãs do ES entre os anos de 2009 e 2011, foram analisadas em conjunto com informaçôes colhidas em três entrevistas semiestruturadas com profissionais da Gerência de Assistência Farmacêutica. Constatou-se expressivo aumento no consumo do medicamento via SUS no período analisado, com distribuição assimétrica entre as oito Farmácias Cidadãs do estado. Tais dados destacam a necessidade de uma análise cuidadosa, atenta aos múltiplos aspectos que interferem tanto na constituição do diagnóstico quanto na demanda por seu tratamento, principalmente no que tange ao acompanhamento da dispensa pública do metilfenidato. O estudo se faz fundamental a fim de embasar a formulação de políticas e o funcionamento de serviços voltados para o TDAH, no contexto da saúde pública.
1 Professora do Programa de Pós-graduação em Psicologia Institucional, Departamento de Psicologia da Universidade Federal do Espírito Santo, Vitória, Brasil. Endereço eletrônico: calimanluciana@ gmail.com

${ }^{2}$ Mestranda do Programa de Pós-graduação em Psicologia Institucional (PPGPSI/UFES), Vitória, Brasil. Endereço eletrônico: ndomitrovic@ gmail.com 
Este artigo resulta de pesquisa realizada entre 2010 e 2012, que visou investigar a dispensa pública do cloridrato de metilfenidato pelo Sistema Único de Saúde brasileiro (SUS). Comercializado no Brasil sob os nomes Concerta ${ }^{\circledR}$ ou Ritalina ${ }^{\circledR}$, o metilfenidato é o psicoestimulante mais consumido no mundo, superando todos os outros somados (ITABORAHY, 2009; ONU, 2011). Atualmente, encontra-se disponível no mercado em apresentações de liberação imediata, cujo efeito é de curta duração, ou de liberação prolongada, que se mantém durante mais tempo no organismo. O metilfenidato é o principal medicamento prescrito para o tratamento do transtorno do déficit de atenção/hiperatividade (TDAH), sendo indicado também para narcolepsia. (ANVISA, 2009; 2012; CALIMAN, 2006; ITABORAHY, 2009; LIMA, 2005; NOVARTIS, s/d). A investigação realizada tem como pano de fundo analítico os processos atuais de farmacologização e medicalização da vida, e neles a expansão do diagnóstico de TDAH e do consumo de metilfenidato.

A indústria farmacêutica moderna teve início no século XIX, quando vários compostos químicos, incluindo alcaloides como morfina, estriquinina, quinina, nicotina e cocaína, foram isolados e produzidos em massa. Mas os maiores desenvolvimentos ocorreram durante e após a Segunda Guerra Mundial. Descobriu-se a penicilina; um número amplo de antibióticos passou a ser comercializado; surgiu a estreptomicina para tratar a tuberculose; contraceptivos, anti-histamínicos, tranquilizantes, anfetaminas, diuréticos e muitos outros compostos passaram a fazer parte de nosso cotidiano (JENKINS, 2010; PETRYNA; LAKOFF; KLEINMAN, 2006).

Cerca de 50 anos depois, em 2003, os gastos globais com a indústria farmacêutica chegaram a quase 500 bilhões de dólares, sendo que quase a metade desta quantia foi atribuída ao Canadá e EUA (PETRYNA; LAKOFF; KLEINMAN, 2006). Por trás deste número, inúmeros dilemas morais e econômicos foram criados. $\mathrm{O}$ consumo de medicamentos, a partir da segunda metade do século XX, aumentou significativamente devido ao fortalecimento do paradigma biomédico, ao crescimento da indústria farmacêutica, à ampliação do acesso aos medicamentos, além da intensificação dos processos de mercantilização da saúde e medicalização da sociedade (POLI; CAPONI, 2007). O medicamento foi transformado na principal tecnologia médica moderna em um momento no 
qual o sofrimento humano e as insatisfações cotidianas têm sido patologizadas e medicalizadas (ROSEMBERG, 2002; ROSE, 2006; CONRAD, 2006). Assim, a discussão sobre medicamentos ultrapassa o campo biomédico, gerando também implicações sociais, econômicas, antropológicas e epidemiológicas (JENKINS, 2010; PETRYNA; LAKOFF; KLEINMAN, 2006).

Para Jenkins (2010), todos nós, em alguma dimensão, estamos nos tornando pessoas farmacológicas, inseridas em um imaginário farmacológico. Muitas das questôes acima apontadas perpassam toda indústria farmacêutica e o consumo de medicamentos, mas os psicotrópicos e a indústria psicofarmacológica possuem sua especificidade, devido à ação de tais medicamentos no cérebro, às experiências resultantes e seus significados culturais. $\mathrm{O}$ cérebro é simbolicamente sentido e considerado a sede da alma, da mente, do eu. Mas em que extensão e de que forma somos mais ou menos moldados pela lógica farmacológica global é algo a ser interrogado, analisado, pesquisado e problematizado.

A autora destaca que não se trata de uma relação de dominação da experiência singular de cada um por uma lógica global que a todos captura da mesma forma e com a mesma força (JENKINS, 2010). No encontro entre uma racionalidade farmacológica global e a experiência local de tomar medicamentos, resistências, incorporações, apropriações ativas se dão; por isso a necessidade de uma perspectiva antropológica na investigação dos processos de expansão do consumo de fármacos. Neste artigo importa investigar, portanto, no que tange ao aumento crescente do consumo de metilfenidato e a expansão do diagnóstico de TDAH, quais processos de apropriação, incorporação e resistências à lógica farmacológica global estão em curso no Brasil, especificamente no Espírito Santo, principalmente no que concerne às políticas públicas de Assistência Farmacêutica voltadas para esse transtorno.

No Brasil, o Ministério da Saúde não inclui o metilfenidato nas suas listas padronizadas de dispensa de medicamentos via Sistema Único de Saúde (SUS), tais como a Relação Nacional de medicamentos essenciais (RENAME), a Portaria $n^{\circ}$ 2.981/GM/MS e suas atualizaçôes. Embora não seja uma obrigatoriedade, cada secretaria estadual e municipal possui relativa autonomia para definir listas próprias que contemplem suas especificidades locais, elencando medicamentos que não estejam nas predefinições nacionais de dispensação pública. Tal medida garante que alguns medicamentos, que até então só poderiam ser acessados pela população via processos judiciais contra o Estado, sejam solicitados por meio de 
processos administrativos nas Farmácias do SUS estaduais, "padronizando" o fluxo de solicitação e dispensa do mesmo. Assim, podemos definir que existem basicamente duas vias de acesso a medicamentos de forma gratuita via SUS. Uma delas é a via judicial, ou seja, sua efetuação ocorre por meio de processos judiciais, geralmente fundamentados no artigo quinto da Constituição brasileira, que define que a saúde é direito do cidadão e dever do Estado. A segunda forma, que muitas vezes é mais lenta, mas menos custosa para os cofres públicos, está na via administrativa. Ou seja, trata-se do processo padrão estabelecido pela política de Assistência Farmacêutica, que, uma vez cumpridos os trâmites burocráticos, dispensa os medicamentos padronizados, seja na lista nacional (RENAME), estadual ou até mesmo municipal.

Desta forma, a Secretaria Estadual de Saúde do Espírito Santo (SESA) inclui o metilfenidato entre os medicamentos a serem fornecidos a sua população por investimento do Tesouro do estado, ao contemplá-lo em sua Relação Estadual de Medicamentos Essenciais e Excepcionais (REMEME) desde sua criação, em 2007 (ESPÍRITO SANTO, 2007). Ou seja, a partir de então, o metilfenidato passa a ser um medicamento padronizado no âmbito da política farmacêutica capixaba, podendo ser solicitado regularmente em suas Farmácias Cidadãs Estaduais.

Sem dúvida, há uma dimensão econômica e política por trás da expansão global da indústria farmacêutica que atinge todos nós, direta e indiretamente, interferindo na definição do que compreendemos por saúde e doença, das terapêuticas privilegiadas para lidar com nossos mal-estares, do que deve ou não ser alvo de nossas políticas de saúde. No Livro Global pharmaceuticals: ethics, markets, practices, Petryna, Lakoff e Kleinman (2006) apresentam em detalhes os processos que fazem com que certos medicamentos e não outros, certas doenças e não outras, se tornam alvo de interesse e desenvolvimentos da indústria farmacêutica. Enquanto cresce o investimento em antidepressivos, outro nicho é deixado de lado, menos rentável, mas talvez mais urgente. Sabemos que todo o continente africano sofre as consequências dessa política e que muitas doenças infecciosas e epidêmicas poderiam ser erradicadas se houvesse investimento no desenvolvimento e distribuição de medicamentos para tratá-las ou preveni-las. Mas quem define o que é urgente, o que é necessidade de saúde, o que é prioridade nas políticas globais e locais?

Este trabalho visou investigar o processo de padronização da dispensa do metilfenidato nas políticas públicas de assistência farmacêutica do ES, bem 
como a avaliação que a gestão da assistência farmacêutica faz sobre tal medida, após quatro anos de dispensação. Além disso, foi traçado o perfil da dispensação pública deste medicamento no Espírito Santo, identificando as especificidades regionais no que se refere à dispensa do medicamento, bem como sua variação numérica ao longo dos anos. Tal investigação visou criar subsídios técnicos e teóricos para a avaliação e análise das políticas públicas de saúde voltadas para o TDAH, especialmente no que tange às políticas da assistência farmacêutica.

Frequentemente descrito como uma desordem global do comportamento, o TDAH se caracteriza pela tríade sintomatológica composta por desatenção, hiperatividade e impulsividade (BARKLEY, 1998; ROHDE et al., 2004; FARAONE, 2003). Os sistemas classificatórios atualmente utilizados, a CID 10 e o DSM IV, apresentam não somente nomenclaturas diferentes (transtorno de déficit de atenção/hiperatividade ou TDAH no DSM IV e transtornos hipercinéticos na CID 10), mas também certas especificidades que merecem especial atenção. Embora ambos os manuais possuam critérios diagnósticos semelhantes, pode-se considerar que os da CID são mais exigentes, uma vez que impõem como condição a existência mútua dos quadros de desatenção e hiperatividade para que se conclua o diagnóstico de transtorno hipercinético. Por sua vez, o DSM IV traz a possibilidade da classificação do transtorno em três subtipos: TDAH com predomínio de sintomas de desatenção, TDAH com predomínio de sintomas de hiperatividade/impulsividade e o TDAH combinado, no qual coexistem os sintomas dos dois subtipos anteriores.

No Brasil, embora os códigos da CID sejam estabelecidos como padrão para solicitação de procedimentos no SUS, os critérios do DSM IV têm ganhado relevância, principalmente após a tradução e validação do questionário SNAP IV, escala de identificação e classificação do TDAH e transtorno desafiador opositor (TDO) (MATTOS et al., 2006).

Apesar de ser aclamado como um dos diagnósticos psiquiátricos atualmente mais estudados no campo biomédico (BARKLEY, 1998; ROHDE et al., 2000, 2004; FARAONE, 2003), o TDAH também tem sido descrito como um diagnóstico controverso (CONRAD, 2006; DUPANLOUP, 2004; RAFALOVICH, 2002; ROSE, 2006; ROSEMBERG, 2002; SINGH, 2006, 2007). Dados sobre o número de sujeitos diagnosticados nos últimos anos e o alarmante aumento do consumo de metilfenidato em várias regiôes do mundo deflagram a necessidade da análise 
cautelosa acerca do diagnóstico. Em certo sentido, o TDAH poderia ser analisado como uma desordem sem fronteiras (ROSE, 2006) - um diagnóstico psiquiátrico que parece não possuir limites internos nem externos. Desde sua constituição, na década de 1970, presenciamos um processo crescente de expansão da categoria. Antes considerado uma desordem transitória e infantil, que raramente alcançava a adolescência, o TDAH é agora descrito como um transtorno psiquiátrico que pode perdurar por toda a vida do indivíduo - um quadro incurável. Uma vez visto como a causa para o baixo desempenho escolar, o transtorno passou a ser uma explicação biológica "plausível" para as dificuldades da vida, sejam elas acadêmicas, profissionais, emocionais, familiares e, mesmo, sexuais (JOFFE, 2005; MATTOS, 2005; WEISS; MURRAY, 2003).

É importante ressaltar que, em 1998, o Instituto Nacional de Saúde Americano (National Health Institutes - NHI, 1998) publicou um documento intitulado Consensus Development Statement on Diagnosis and Treatment of Attention Deficit Hyperactivity, no qual fica explícita a falta de consenso no meio científico a respeito do transtorno, não havendo real comprovação de sua causalidade orgânica e, mais especificamente, cerebral (NHI, 1998). Recentemente, um trabalho realizado pelo instituto canadense Hasting Center de pesquisa em Bioética veio atualizar as questôes levantadas pelo documento publicado uma década antes pelo NHI, demonstrando que até o momento atual, especialistas discordam sobre o diagnóstico do TDAH e seu tratamento (PARENS; JOHNSTON, 2009).

Ambos os documentos destacam a dificuldade de se estabelecer limites objetivos entre o TDAH, outras desordens do comportamento e o comportamento normal. Isso se dá, em grande parte, por não existirem marcadores biológicos definitivos que acusem sua ocorrência. Seu diagnóstico se faz, portanto, por meio de "investigação médica, neuropsicológica, educacional e social" (NOVARTIS, s/d, p.8), na qual se avalia clinicamente o prejuízo que os sintomas provocam na vida do sujeito, com apoio nos critérios definidos nos manuais diagnósticos DSM IV ou CID 10 (CALIMAN, 2006; GENRO, 2008; ROHDE et al., 2000; ROHDE; HALPERN, 2004; NOVARTIS, s/d).

Com relação ao tratamento, o documento produzido pelo NHI (1998) revela que pesquisas têm sido direcionadas para o desenvolvimento de medicamentos e intervenções psicossociais. No entanto, são raros os estudos que investigam os riscos e benefícios de tais intervenções no longo prazo. Neste sentido, não 
é possível afirmar que as terapêuticas utilizadas tenham impacto positivo ou negativo nos desempenhos educacionais e profissionais, queixas principais dos indivíduos com TDAH. O caráter não conclusivo do diagnóstico e de seu tratamento é ainda mais destacado no caso do subtipo desatento, principalmente no que se refere a adolescentes e adultos. $\mathrm{O}$ NHI atesta, ainda, que pacientes com problemas diversos de desatenção e não diagnosticados com TDAH respondem positivamente ao medicamento. Neste caso, alerta-se para o risco do uso não médico do metilfenidato, fenômeno que tem sido comum entre jovens universitários de diversos países, inclusive no Brasil (ANVISA, 2009; BARROS, 2011).

Além dos fatores acima mencionados, que indicam o caráter inconclusivo e por vezes polêmico do TDAH, soma-se a atual tranformação que vem sofrendo o tratamento medicamentoso do transtorno, tanto quanto a sua complexidade quanto a sua duração. Até o ano 2000, a maioria das crianças diagnosticadas com TDAH era tratada com medicamentos de liberação imediata, apenas no período escolar e durante um ou dois anos. Atualmente, muitas crianças já fazem uso das drogas de liberação prolongada, de forma a permanecer sob seu efeito durante e depois o período escolar. Segundo Parens e Johnston (2009), o posicionamento recente de um número considerável de especialistas se centra no uso cada vez mais precoce da medicação e "pelo tempo que for necessário" (p. 2, tradução nossa), de forma que a tendência atual é que os tratamentos se iniciem cada vez mais cedo na vida das crianças e se prolonguem por tempo indiscriminado. Esta última tendência é justificada pela expansão da categoria diagnóstica que passou a abarcar também indivíduos adultos (CALIMAN, 2006).

Todos esses aspectos ganham maior dimensão e importância diante do aumento mundial do consumo do metilfenidato. Segundo dados da ONU (2011), o consumo e produção deste psicoestimulante vêm aumentando mundialmente de forma significativa desde os anos 1990. No início da década, o volume total produzido mundialmente foi de 2,8 toneladas. Passados dez anos, em 2000 a produção atingiu um volume sete vezes maior, chegando a 16 toneladas. Em 2010, a estatística atingiu a marca de 43 toneladas. No mesmo relatório, a ONU aponta o metilfenidato como o psicoestimulante mais utilizado no mundo. Enquanto em 2001, a produção de metilfenidato foi responsável por 22,5\% do volume total de estimulantes fabricados no planeta, apenas nove anos depois, em 2010, sua contribuição subiu para 56\%. 
No Brasil, o uso de metilfenidato também tem crescido ao longo dos anos. Tendo sua comercialização aprovada desde 1998 (ANVISA, 2012), o consumo nacional em 2000 era de $23 \mathrm{~kg}$. Segundo documento da ONU, apenas seis anos depois, o Brasil fabricava $226 \mathrm{~kg}$ e importava outros $91 \mathrm{~kg}$ do medicamento (LIMA, 2005). Mais recentemente, a ANVISA divulgou que em 2011 as vendas de metilfenidato chegaram a mais de $413 \mathrm{~kg}$ do produto (ANVISA 2012). O gasto estimado das famílias brasileiras com o produto, nesse mesmo ano, foi de $\mathrm{R}$ \$ 28,5 milhôes, o que equivale a um valor de $\mathrm{R} \$ 778,75$ por cada mil crianças com idade entre seis e 16 anos.

$\mathrm{O}$ metilfenidato tem ganhado destaque nos últimos estudos da ANVISA referentes ao consumo nacional de medicamentos por via particular, gerados a partir do Sistema Nacional de Gerenciamento de Produtos Controlados (SNGPC/ ANVISA). O primeiro relatório do SNGPC, divulgado em 2009, tinha por objetivo principal produzir dados sobre a prescrição e consumo nacional de anfetaminas anorexígenas, já que o Brasil fora apontado pela ONU como o maior consumidor de anfetaminas com finalidade emagrecedora (ANVISA, 2009). No entanto, o metilfenidato foi também incluído no relatório, devido a seu uso maciço:

[...] com uso muito difundido, [o metilfenidato] vem sendo foco de vários estudos e questionamentos quanto ao seu uso massivo e efeitos secundários. Sua utilização já está ocorrendo, por exemplo, entre empresários e estudantes para emagrecimento e até para uso recreacional, na forma triturada como pó ou diluído em água para ser injetado. (ANVISA, 2010).

Em 2012, a agência divulgou um boletim voltado exclusivamente à questão do metilfenidato, descrevendo sua prescrição e consumo no Brasil, nos anos de 2009 a 2011, apontando "possíveis distorções na utilização de metilfenidato" (ANVISA 2012). Como já mencionado, os estudos da ANVISA se baseiam em relatórios gerados pelo SNGPC, que consiste em um sistema informatizado alimentado por farmácias privadas de todo o país. Desta forma, nesses estudos não comparece a questão da dispensação pública, cujo rastreamento e avaliação não têm sido feitos de forma sistematizada pela ANVISA. Além disso, a literatura científica brasileira sobre o medicamento não diferencia o consumo via particular de sua dispensação pública.

$\mathrm{O}$ transtorno de déficit de atenção e hiperatividade (TDAH), associado ao consumo crescente de metilfenidato, tem sido descrito como um dos principais diagnósticos envolvidos no processo de medicalização da vida. Entre algumas das questôes apontadas como problemáticas, estão o número excessivo de diagnósticos 
em crianças e adultos; a patologização de questões que são de ordem educacional e social; e a expansão do uso não médico do metilfenidato com o objetivo de melhorar a performance cognitiva ou para fins recreativos (CALIMAN, 2008). Assim, dado o caráter polêmico e controverso do TDAH e seu tratamento medicamentoso, acredita-se que o crescimento na produção e no consumo do metilfenidato no Brasil, em tão pouco tempo, tornou imprescindível a compreensão dos usos do medicamento em território nacional, principalmente no que diz respeito à sua dispensação pelo SUS. Espera-se, portanto, que as análises oferecidas sobre o Espírito Santo possam estimular a investigação das políticas públicas da assistência farmacêutica voltadas para o TDAH em outros estados e municípios.

\section{Metodologia}

Para uma melhor compreensão do panorama brasileiro das Políticas Públicas de Assistência Farmacêutica voltadas para o TDAH, foi construído um mapa da dispensação pública do metilfenidato no país, diferenciando quais estados possuíam listas estaduais de medicamentos padronizados para a dispensação via SUS e, entre elas, quais incluíam o cloridrato de metilfenidato. Tais informações foram coletadas por meio de ligaçóes telefônicas junto às assistências farmacêuticas estaduais, assim como em consultas em seus respectivos sites oficiais.

Com o intuito de investigar o processo de inclusão do metilfenidato na REMEME e, a partir de então, o posicionamento da Gerência da Assistência Farmacêutica do Espírito Santo (GEAF) sobre sua dispensação, foram realizadas entrevistas semiestruturadas com três profissionais da GEAF. Todas as entrevistas foram gravadas em áudio e posteriormente transcritas para então serem analisadas. Em seguida, mapearam-se as características da demanda e da dispensação pública de metilfenidato no Estado do Espírito Santo. Para tanto, foram coletados os seguintes dados: número de abertura de processos de solicitação, entre 2009 e 2011, nas oito Farmácias Cidadãs Estaduais do Espírito Santo; quantidade média de comprimidos dispensados por mês em cada farmácia; gasto anual da Secretaria Estadual de Saúde com o medicamento, de 2008 a 2011.

Após o tratamento e análise dos dados, os resultados da pesquisa foram apresentados, discutidos e validados em reunião com a GEAF e coordenadores das oito Farmácias Cidadãs do Estado do Espírito Santo. Esta pesquisa se realizou sob aprovação do Comitê de Ética em Pesquisa com Seres Humanos da Secretaria Estadual de Saúde do ES, conforme a Resolução no 196/96 do CNS. 


\section{A dispensa pública do metilfenidato no Brasil}

A pesquisa revelou que, no primeiro semestre de 2010, dez das 27 assistências farmacêuticas do país não possuíam listagens estaduais de medicamentos padronizados para dispensação pública via SUS, adotando integralmente, portanto, as definiçóes nacionais. O Espírito Santo, por sua vez, se destaca entre os 11 estados que possuem elencos próprios de medicamentos padronizados, estando ainda entre os únicos quatro que incluem o metilfenidato.

Figura 1. Mapeamento da situação das Assistências Farmacêuticas no Brasil até abril/2011. As AF dos estados "Sem informação" são aquelas que houve insucesso tanto no contato telefônico quanto na pesquisa em sites oficiais.

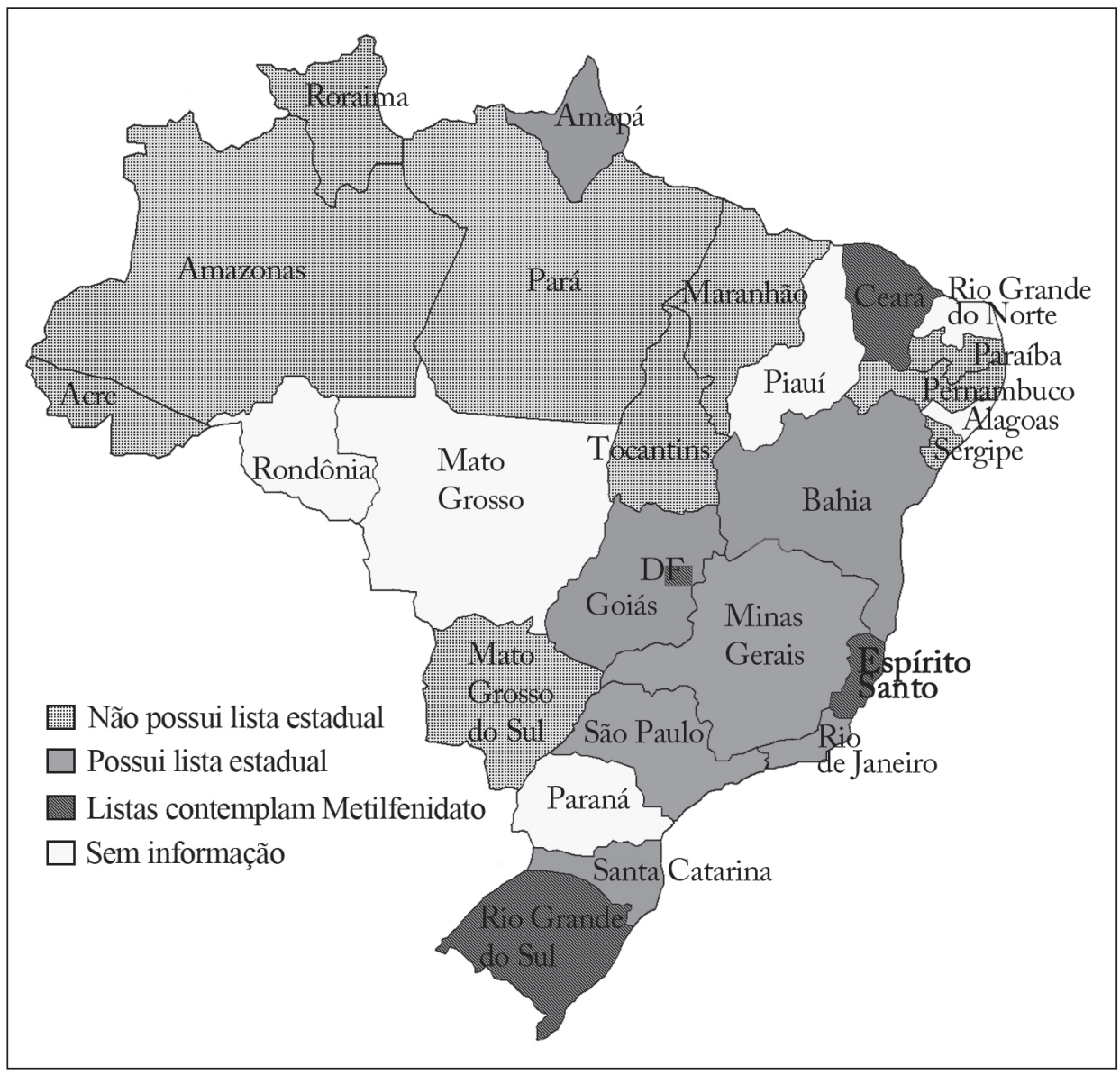


É importante considerar que tais dados não trazem informações conclusivas sobre a dispensa pública do metilfenidato no Brasil, mas indicam como estão organizadas as assistências farmacêuticas estaduais. $\mathrm{O}$ fato de o metilfenidato não estar padronizado em certos estados não garante que sua dispensa pública não ocorra. Tal afirmação se explica, em parte, pela possibilidade da existência de listagens municipais que contemplem o medicamento. O Estado de São Paulo, por exemplo, possui lista de medicamentos estaduais, mas não inclui nela o metilfenidato. A cidade de Santos, no entanto, dispensa o medicamento no nível municipal (SANTOS, 2009). Além disso, como já exposto, a dispensa pública pode ocorrer via abertura de processos judiciais pelos usuários, para a solicitação de medicamentos não padronizados. As entrevistas realizadas revelaram que no Espírito Santo, antes de 2007, a demanda pelo metilfenidato era motivo frequente de processos judiciais impetrados contra o Estado, sendo este um fator motivador para a inserção do medicamento no elenco padronizado.

A inserção do metilfenidato na REMEME em 2007 ocorreu, portanto, entre outros motivos, sob influência dos processos de judicialização da assistência farmacêutica. De acordo com o relato de um de seus profissionais, em 2007 o cotidiano da GEAF já era marcado pelo impacto da judicialização. Nesse mesmo ano, tal situação culminou com o mandato de prisão do secretário de Saúde, tendo como pivô o medicamento Avastin ${ }^{\circledR}$, solicitado para o tratamento de um tumor cerebral. Tal evento ganhou projeção nacional, sendo divulgado pelo Portal de Notícias G1 (acesso em 20 dez. 2011).

Além dos processos judiciais, a entrada do metilfenidato na REMEME foi impulsionada por uma solicitação formal de um médico especialista. Diante dessas demandas e após uma revisão da literatura sobre as evidências científicas que aprovavam o uso médico do metilfenidato, o medicamento passou a constar na lista de medicamentos estaduais, na medida em que a comissão responsável acreditava que "tendo o diagnóstico bem feito, é um recurso terapêutico que pode melhorar, promover qualidade de vida para ela [criança]" (Entrevistado 1).

No entanto, alarmada com o aumento de solicitações do medicamento e com problemas nos critérios de sua dispensação, dois anos após sua inclusão na REMEME, a GEAF decidiu rever o protocolo clínico que regularizava a dispensa pública do metilfenidato. O protocolo regularizador da dispensação pública do metilfenidato no Espírito Santo encontra-se, portanto, em sua segunda versão, homologada em 
setembro de 2010 (ESPÍRITO SANTO, 2010). O primeiro protocolo data de 2007, ano em que a REMEME entrou em vigor. Entre as motivações para a revisão do protocolo, os entrevistados apontam ainda o grande número de processos solicitando o metilfenidato de liberação prolongada, em detrimento do de liberação imediata, além de expressiva demanda por parte de adultos.

A leitura comparativa entre os dois protocolos evidencia um movimento de abertura dos critérios de dispensação do medicamento, principalmente no que se refere à população atendida: o CID F90.1 (Transtorno hipercinético de conduta) é incluído junto ao F90.0 (transtorno hipercinético-perturbação da atividade e atenção) na classificação de usuários habilitados a solicitarem o medicamento, assim como usuários adultos passam a ser também aceitos, já que antes o medicamento era dispensado somente para o público infantil. Além disso, no segundo protocolo, o acompanhamento psicoterápico deixa de ser exigência para a retirada do medicamento. Tal decisão é motivada, segundo um dos entrevistados, não pela negação de sua importância, mas pela dificuldade de acesso à psicoterapia por parte dos usuários na rede pública. Em duas das entrevistas, a importância de um acompanhamento cuidadoso do diagnóstico e do tratamento foi destacada:

A análise inicial que a gente faz é uma análise do ponto de vista do tratamento. E este assunto é interessante porque o tratamento do déficit de atenção não é só o medicamento, né? Não é só ingerir o comprimido. Ele passa por um tratamento, tem que ser mais contextualizado, multiprofissional. É uma doença que tem haver com comportamento. E isso foi uma coisa que preocupou a gente na época. Mas a limitação é que a gente é uma gerência farmacêutica, a gente não dá conta, a gente não tem como intervir em toda a rede de saúde, que é muito ampla, tem outras gerências envolvidas. A gente é responsável só pelo medicamento. E isto foi um problema que a gente teve, porque na época que foi feita a padronização a gente viu que era importante o acompanhamento psicoterapêutico. Mas a gente esbarra na estrutura insuficiente da rede de saúde que não oferece este tipo de tratamento (...) então a gente reviu este aspecto. (Entrevistado 1).

As pessoas hoje são muito mal atendidas, em todos os lugares. Fazer esses diagnósticos e acompanhá-los é muito importante porque o diagnóstico é muito complicado de ser feito. Reflete em várias coisas na vida da pessoa. Não é só uma coisa pontual que acaba ali, faz parte da vida dela. (Entrevistado 2)

No novo protocolo, os medicamentos de duração prolongada passaram a ser também padronizados, mas sob exigências mais rígidas para sua dispensação, como comprovação da dificuldade de adesão ao tratamento de liberação imediata. 
Então o seguinte, se o paciente fez uso do metilfenidato $10 \mathrm{mg}$ durante três meses com

a posologia de pelo menos três tomadas diárias e este indivíduo apresentou dificuldade de adesão ao tratamento, com o comprometimento da eficácia, comprovado por laudo médico, a gente entende que está justificado que ele faça uso de um medicamento que permita menos tomadas, que facilita a adesão. Fora isso não. A gente entende que [o uso do medicamento] fica mais racional desta forma. (Entrevistado 1).

Assim, o novo protocolo, embora mais abrangente em termos de público atendido e apresentações disponíveis, sugere, por outro lado, uma tentativa de maior regulação da dispensação, principalmente diante da constatação do aumento "explosivo" dos pedidos do medicamento de liberação prolongada e da pressão pela via judicial,

A demanda por este tratamento se tornou explosiva. Aí traz uma preocupação com relação ao diagnóstico (...). A gente tinha aprovado duas apresentações do metilfenidato: de liberação imediata, que é Ritalina ${ }^{\circledR}$, e a de liberação prolongada que é o Concerta. Com o passar do tempo foi ver com maior cuidado, né? A gente viu que não tinha muito fundamento a gente oferecer o Concerta, porque do ponto de vista da eficácia, ele não é mais eficaz que o de liberação imediata, a única vantagem que a gente observou é a da comodidade fisiológica, de uma tomada diária. (Entrevistado 1).

$\mathrm{Na}$ verdade, [a inclusão da apresentação de liberação prolongada] partiu dessa coisa da judicialização. Acaba tendo tanto processo, a pressão passa a ser externa, sabe? Ameaça a prender o secretário de saúde, essa pressão toda. (Entrevistado 2).

[...] quando o metilfenidato foi incluído na apresentação de 10 e de 20 e a 18 e 54, que é o Concerta, então nós percebemos ao longo dos anos um aumento exorbitante, a gente fez esse acompanhamento né, na prescrição do Concerta. Então nós paramos pra começar a rever. O que está acontecendo? Porque a prescrição tão alta? E começamos a analisar os processos caso a caso. A gente via muito que era comodidade posológica na maioria dos casos. E começamos a fazer também um estudo de custo/benefício. Porque o concerta e não a Ritalina LA? Também é liberação prolongada. E a gente começou e cada hora ia surgindo novos dados. A gente começou a fazer, chamamos a sociedade de Pediatria, a sociedade de Neuro e a de Psiquiatria. E chamamos todos pra cá e conversamos acerca do metilfenidato. Porque a gente começou a ter um gasto que não estava planejado. Então a gente começou a discutir se realmente tinha de ser o Concerta, ou não, todos os pacientes precisam que seja, começamos a discutir com as sociedades médicas. Por isso revemos o protocolo em cima dessa discussão (Entrevistado 3).

Tanto as entrevistas realizadas quanto contatos posteriores com a gestão da GEAF demonstraram que, no Espírito Santo, a dispensação pública do metilfenidato é acompanhada de incertezas, tensões e discussões. Ao falar da situação paradoxal do metilfenidato para a assistência farmacêutica, um profissional da CEFT afirmou: "ruim com ele, pior sem ele". O medicamento é descrito como um recurso importante para o tratamento do TDAH, demandado 
pelas associações médicas e usuários, mas ao mesmo tempo é envolto em polêmicas que alertam para o perigo de seu uso isolado, não acompanhado por outras medidas que abrangeriam a rede pública de saúde e educação. Por outro lado, o próprio diagnóstico de TDAH é descrito como complexo e incerto.

O metilfenidato se constitui enquanto objeto de preocupação para a assistência farmacêutica capixaba, que atesta a necessidade de criação de dispositivos de regulação da dispensa e acompanhamento de seu uso terapêutico. Em reunião de devolutiva, realizada para a apresentação dos dados desta pesquisa à GEAF e aos coordenadores das oito Farmácias Cidadãs do Estado, tal postura de alerta se fez ainda mais presente diante do mapeamento da demanda estadual pelo medicamento. Apesar de constatar o aumento das solicitaçôes do medicamento e este ser um fator de preocupação da GEAF, até então as características desse aumento não tinham sido alvo de estudo pormenorizado.

\section{A demanda e dispensa pública de metilfenidato no Espírito Santo}

Os números abaixo referem-se às aberturas de processos de solicitação de metilfenidato em todas as Farmácias Cidadãs estaduais do Espírito Santo, de 2009 a 2011. Portanto, expressam a demanda pelo medicamento à dispensa pública do estado.

Gráfico 1. Número de processos abertos para a solicitação de metilfenidato em todas as Farmácias Cidadãs estaduais do Espírito Santo, nos anos de 2009, 2010 e 2011 (cada processo foi contado apenas uma vez, com referência à sua data de abertura).

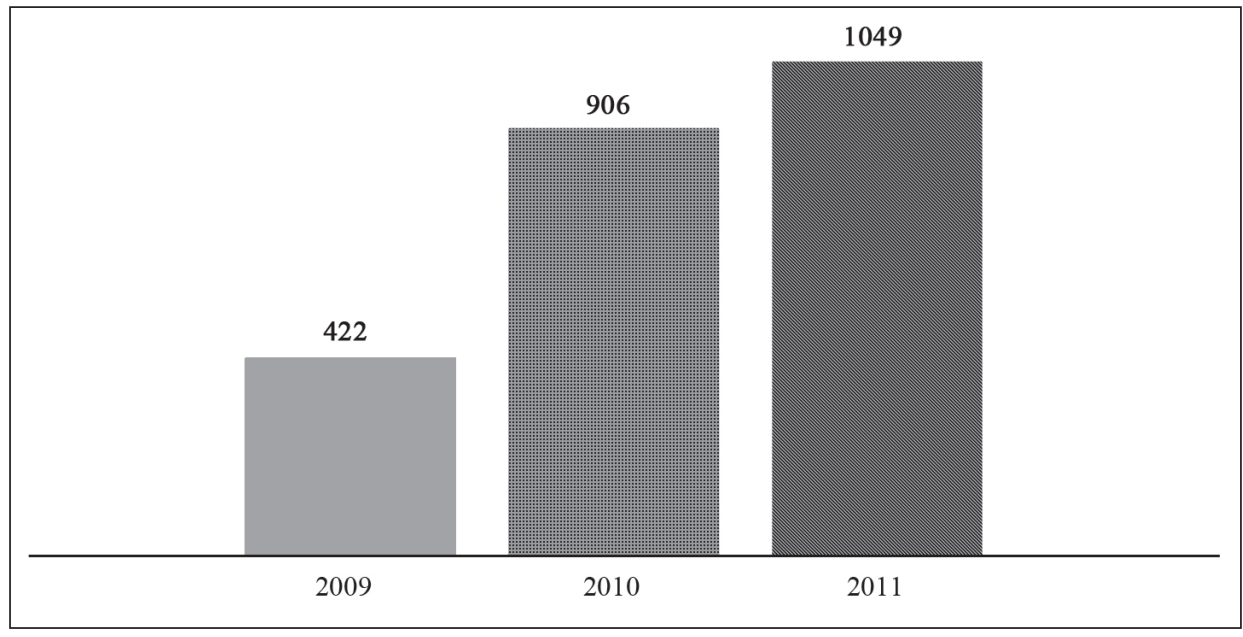


Observa-se que houve aumento crescente na procura pela dispensa pública do metilfenidato no ES entre 2009 e 2011, chegando ao acréscimo de quase duas vezes e meia no último ano em relação ao primeiro. Tal crescimento na demanda vem acompanhado de um robusto aumento dos gastos com o metilfenidato pela secretaria: em 2009, o gasto anual na compra do psicoestimulante totalizou R \$ 1.699.254,20. Dois anos depois, o investimento para sua compra havia sofrido um aumento de $178 \%$, alcançando a cifra de $\mathrm{R} \$ 3.026 .167,80$ (GEAF, mensagem obtida em 24 fev. 2012). No entanto, a mesma configuração não se reproduz em todas as Farmácias Cidadãs Estaduais, quando analisadas separadamente.

\section{Gráfico 2. Número de processos abertos para a solicitação de metilfenidato em cada uma das Farmácias Cidadãs estaduais do Espírito Santo, nos anos de 2009, 2010 e 2011 (cada processo foi contado apenas uma vez, com referência à sua data de abertura).}

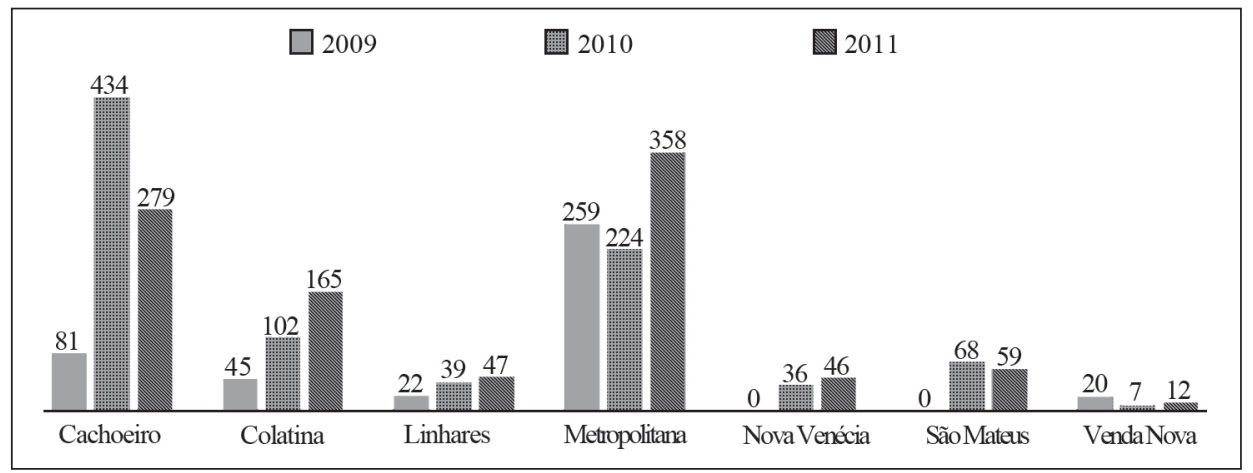

O número de abertura de processos de solicitação de metilfenidato variou de forma significativamente heterogênea nas diferentes localidades do Espírito Santo no período entre 2009 e 2011. A discrepância na demanda nas Farmácias de Cachoeiro do Itapemirim e Metropolitana em relação às demais merece destaque: o número de processos registrados nas duas farmácias representa $69 \%$ da demanda total do estado nos três anos. Em ambas houve crescimento significativo da demanda registrada no ano de 2011 em relação a 2009.

A mesma situação é observada em relação à retirada do medicamento, ou seja, a sua liberação pela GEAF. Ao se fazer a média das liberações no período de 01/06/2011 a 31/08/2011, período de estoque totalmente regular para efeito de comparação, percebe-se que a demanda tem sido, nos dizeres da Coordenação da GEAF, "assustadoramente” maior na Farmácia de Cachoeiro de Itapemirim. 

do ES, no período de 01/06/2011 à 31/08/2011 (GEAF, mensagem obtida em jan. 2012).

\begin{tabular}{|c|c|}
\hline FARMÁCIA & MÉDIA DE COMPRIMIDOS/MÊS \\
\hline Cachoeiro do Itapemirim & 12.000 \\
\hline Metropolitana & 4.400 \\
\hline Vila velha & 2.100 \\
\hline Colatina & 2.270 \\
\hline Linhares & 700 \\
\hline Venda Nova do Imigrante & 400 \\
\hline São Mateus & 2.040 \\
\hline Nova Venécia & 220 \\
\hline
\end{tabular}

Confrontados com os dados acima, tanto GEAF quanto coordenadores das farmácias cidadãs descreveram como alarmante e preocupante o número elevado de processos advindos das farmácias de Cachoeiro de Itapemirim e Metropolitana, e destacam que a discrepância regional das solicitaçôes de metilfenidato coloca em questão o próprio diagnóstico de TDAH.

Em Cachoeiro de Itapemirim, onde nós tivemos um aumento de demanda muito grande, isso leva a gente a se preocupar. Principalmente porque é uma demanda que é muito diferente de outras regiōes do Estado, por exemplo. Se a gente pensar que a doença em geral vai ter uma prevalência mais homogênea, é estranho você pensar que em determinadas região você tenha um número tão grande de casos. Então, o diagnóstico não está correto... e é isso. E por se tratar de uma doença que envolve comportamento o diagnóstico é subjetivo, né?! [...] (Entrevistado 1).

Os dados da pesquisa revelam diferenças numéricas regionais na solicitação e dispensa do metilfenidato que só poderiam ser mais bem analisadas através de pesquisas de cunho antropológico e sócio-histórico, que investigassem as especificidades de cada região. Sabemos, no entanto, que a cidade de Cachoeiro de Itapemirim tem sido historicamente marcada por processos de medicalização que vão na contramão da Política de Saúde Mental Nacional e do Movimento da Reforma Psiquiátrica, tornando-se solo fértil para a expansão dos processos de farmacologização da vida. 
A variação no consumo do medicamento no Espírito Santo encontra também paralelo com os dados nacionais, advindos do relatório sobre a rede privada de farmácias publicado pela ANVISA em 2010:

Gráfico 3. Consumo de metilfenidato no Brasil, por via particular, per capita no ano de 2009 (ANVISA, 2010).

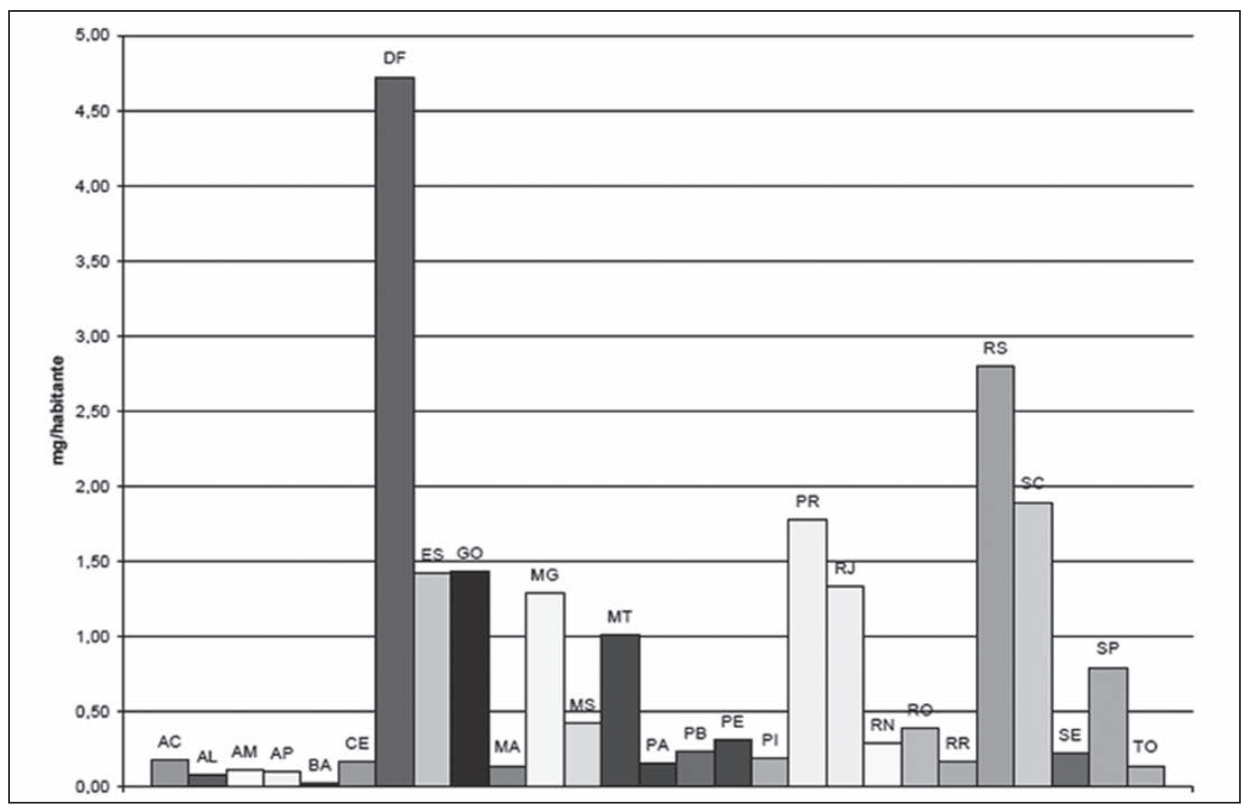

Segundo a ANVISA (2010; 2012), os estados do Brasil apresentam perfis variados de consumo do medicamento. Para a agência, tal fato indica a necessidade de medidas adaptadas a cada caso, após estudo epidemiológico, tendo em vista a diminuição do uso abusivo.

No Espírito Santo, diante do panorama acima traçado, a GEAF tem buscado alertar os médicos capixabas para que ao menos as exigências do protocolo clínico, necessário à solicitação do metilfenidato, sejam observadas. Semanas após a reunião de devolutiva da pesquisa com os coordenadores das farmácias cidadãs estaduais, foi encaminhada pela GEAF, ao Conselho Regional de Medicina do ES, uma nota técnica solicitando atenção especial aos critérios de diagnóstico definidos no protocolo (CRM-ES, mensagem obtida em 3 mar. 2012). E ainda, há casos no estado em que crianças com menos de cinco anos 
estão sendo medicadas com metilfenidato, o que viola claramente os critérios de dispensação do medicamento (GEAF-ES, mensagem obtida em 5 mar. 2012).

A diferença regional do consumo do metilfenidato encontrada no âmbito brasileiro acompanha os dados sobre a prevalência do diagnóstico de TDAH que variam amplamente entre os países e também no interior de cada nação nas quais dados epidemiológicos se encontram disponíveis (ROSE, 2006; SINGH, 2006). A literatura apresenta taxas de prevalência bastante variáveis, desde $1 \%$ até próximo de 20\%, (FARAONE et al., 2003; GENRO, 2008). Pesquisas a respeito da prevalência de TDAH em território brasileiro são ainda escassas e, segundo a revisão de literatura realizada, até o momento não trazem coletas simultâneas em diferentes regiōes. Entretanto, quando comparadas entre si, estas apontam também divergências. Em estudo realizado em escolas públicas do Rio de Janeiro, a prevalência encontrada foi de 13\% (FONTANA et al., 2007), enquanto em outro, realizado na cidade de Salvador, encontrou-se a taxa de 5,3\% para uma alta probabilidade do subtipo desatento, $2 \%$ para o subtipo hiperativo/impulsivo e apenas 0,6\% para o subtipo combinado (FREIRE; PONDÉ, 2007). Outra pesquisa, realizada também no ano de 2007 na cidade do Rio de Janeiro, encontrou, entre alunos do Colégio de Aplicação da Universidade Federal do Rio de Janeiro, 8,6\% de prevalência de TDAH (PASTURA; MATTOS; ARAÚJO, 2007).

Existem diversas linhas de análise a respeito de tal discrepância, que resultam em posições críticas muito heterogêneas. Estas oscilam entre uma denúncia de banalização do transtorno e o excesso de diagnósticos em alguns países e regiōes (COLLARES; MOYSES, 2010) e a defesa de que, em certos lugares, o TDAH seria ainda subdiagnosticado (ROHDE, 2004; FARAONE, 2003), por efeito de informação insuficiente de alguns profissionais da saúde e educação sobre a doença, "e/ou equívocos quanto a seu impacto negativo sobre crianças" (FARAONE, 2003, p. 104, tradução nossa).

Em alguns casos, a discrepância acima descrita é vista pelos autores como sinal da interferência de questões culturais, sociais, políticas e subjetivas na definição do diagnóstico de TDAH e na opção por seu tratamento medicamentoso (CONRAD, 2006; PARENS; JOHNSTON, 2009; RAFALOVICH, 2002; ROSEMBERG, 2002). Outros destacam a influência dos critérios e ferramentas diagnósticas adotadas (FREIRE; PONDÉ, 2005; GENRO, 2008; PASTURA; MATTOS; ARAÚJO, 2007), como se tal explicação anulasse a anterior. Por outro lado, 
pode-se considerar que esta justamente ressalta a dimensão cultural e subjetiva do processo diagnóstico, no qual fatores como, por exemplo, uma tolerância maior ou menor em relação aos comportamentos desatentos e hiperativos, em determinado contexto, assim como a pressão social e econômica sobre a exigência de sucesso acadêmico e profissional, podem interferir diretamente no uso das ferramentas diagnósticas. É preciso lembrar, ainda, que a própria existência ou não de políticas educacionais, de saúde e da assistência farmacêutica direcionadas para o TDAH podem interferir na maior ou menor demanda pelo diagnóstico. Em todo caso, os dados apresentados reforçam a importância de se afirmar que o debate em torno do transtorno e seu tratamento permanecem em aberto e que, devido ao caráter polêmico e inconclusivo do TDAH, as políticas públicas voltadas para o transtorno devem ser mais bem analisadas e acompanhadas.

\section{Considerações finais}

Amplamente presente no meio científico e midiático, a discussão em torno do TDAH e o metilfenidato tem ganhado também espaço no âmbito das políticas públicas de saúde brasileiras. A presença do medicamento entre os cinco escolhidos para a composição de detalhado estudo da ANVISA (2010), bem como no interior das políticas de assistência farmacêutica, demonstra tal fato.

Tanto o vertiginoso aumento do consumo do metilfenidato em várias regiōes do mundo quanto a discrepância numérica e regional de seu uso alertam para a importância de uma análise cuidadosa do fenômeno. Uma vez que tal quadro se mostra presente na realidade brasileira, seu estudo se faz fundamental, sobretudo para embasar a formulação de políticas e o funcionamento de serviços no contexto da saúde pública. Como aponta a ANVISA, para tanto é indispensável a análise cuidadosa da realidade de cada região.

Este trabalho visou demonstrar que não somente o uso abusivo ou não médico do metilfenidato deve ser alvo de análise cuidadosa, como também aquele autorizado pela identificação médica e social do diagnóstico de TDAH, uma vez que a fronteira entre este e aquele nem sempre é claramente demarcada. Ao mesmo tempo, destaca-se que tal análise deve se ater aos múltiplos aspectos que interferem tanto na constituição do diagnóstico de TDAH quanto em sua demanda pelos atores envolvidos em sua identificação: médicos, pais, professores, crianças e adultos suspeitos de terem TDAH. ${ }^{1}$ 


\section{Referências}

AGÊNCIA DE VIGILÂNCIA SANITÁRIA. Sistema Nacional de Gerenciamento de Produtos Controlados: resultados 2009. Brasília: Agência Nacional de Vigilância Sanitária, 2010. Disponível em: <http://www.anvisa.gov.br/sngpc/resultados_2009.pdf>. Acesso em: 20 dez. 2011.

AGÊNCIA DE VIGILÂNCIA SANITÁRIA. Prescrição e consumo de metilfenidato no Brasil: identificando riscos para o monitoramento e controle sanitário Boletim de Farmacoepidemiologia do SNGPC, ano 2, n. 2, jul./dez. 2012. Disponível em: <http://www. anvisa.gov.br/sngpc/boletins/2012/boletim_sngpc_2_2012_corrigido_2.pdf >. Acesso em: 06 mar. 2012.

BARKLEY, R.A. Developmental course, adult outcome, and clinic-referred ADHD adults. In: BARKLEY, R. (Org.). Attention Deficit Hyperactivity Disorder: a handbook for diagnosis and treatment. New York: Guilford Press, p. 186-224, 1998.

BARROS, D.; ORTEGA, F. Metilfenidato e aprimoramento cognitivo farmacológico: representações sociais de universitários. Saúde e Sociedade. São Paulo, v.20, p. 350-362, 2011.

CALIMAN, L.V. A biologia moral da atenção: a construção do sujeito (des)atento. 2006. Tese (Doutorado em Saúde Coletiva) - Instituto de Medicina Social, Universidade do Estado do Rio de Janeiro, Rio de Janeiro, 2006.

O TDAH: entre as funções, disfunçôes e otimização da atenção. Psicologia em Estudo, Maringá, v. 13, n. 3, p. 559-566, jul./set, 2008.

COLLARES, C.A.L.; MOYSES, M.A.A. Dislexia e TDAH: uma análise a partir da ciência médica. In: CRPSP; GIQE. (Org.). Medicalização de Crianças e Adolescentes. São Paulo: Casa do Psicólogo, 2010, p. 71-110.

CONRAD, P. Expansion: from hyperactive children to adult ADHD. In:

(Org.).The medicalization of society: on the transformation of human conditions into treatable disorders. Baltimore: Johns Hopkins University, 2006. p.46-69.

CONSELHO REGIONAL DE MEDICINA DO ESPÍRITO SANTO. Enc.: Nota Técnica Prescrição do Medicamento Metilfenidato [mensagem pessoal]. Mensagem obtida por calimanluciana@gmail.com em 3 mar. 2012.

DUPANLOUP, A. L'Hyperactivité Infantile: Analyse Sociologique d'une Controverse SocioMédicale.Tese (Doutorado em Ciências Sociais) - Université de Neuchâtel, Neuchâtel, França, 2004.

ESPÍRITO SANTO (Estado). Relação Estadual de Medicamentos Essenciais e Medicamentos Excepcionais - REMEME. Vitória, 2007. Disponível em: <http://www.saude.es.gov.br/> Acesso em: 02 mar 2010. 
FARAONE S. V. et al. The worldwide prevalence of ADHD: is it an American condition? World Psychiatry, v.2, p.104-113, 2003.

FONTANA, R.S. et al . Prevalência de TDAH em quatro escolas públicas brasileiras. Arq. Neuro-Psiquiatr., São Paulo , v.65, n.1, mar. 2007. Disponível em <http://www.scielo.br/ scielo.php? script $=$ sci_arttext $\&$ pid $=$ S0004-282X2007000100027\&lng $=$ pt $\& \mathrm{nrm}=\mathrm{iso}>$. Acesso em: 30 jul. 2013.

FREIRE, A.C.C.; PONDE, M.P. Estudo piloto da prevalência do transtorno de déficit de atenção e hiperatividade entre crianças escolares na cidade do Salvador, Bahia, Brasil. Arq.Neuro-Psiquiatr., São Paulo, v.63, n.2b, jun. 2005. Disponível em <http://www.scielo. br/scielo.php?script $=$ sci_arttext \&pid $=$ S0004-282X2005000300020\&lng=pt \&nrm =iso $>$. Acesso em 30 jul. 2013.

GENRO, J.P. O gene do transportador de dopamina e a suscetibilidade genética ao transtorno de déficit de atenção/hiperatividade em crianças. Tese (Doutorado em Genética e Biologia Molecular) - Programa de Pós-Graduação em Genética e Biologia Molecular - Universidade Federal do Rio Grande do Sul, Porto Alegre, 2008.

GERÊNCIA ESTADUAL DE ASSISTÊNCIA FARMACÊUTICA (Espírito Santo). Metilfenidato [mensagem pessoal]. Mensagem obtida por < calimanluciana@gmail.com> em 05 mar. 2012.

.Dados metilfenidato [mensagem pessoal]. Mensagem obtida por <calimanluciana@ gmail.com> em 24 fev. 2012.

Informaçôes sobre o metilfenidato [mensagem pessoal]. Mensagem obtida por <calimanluciana@gmail.com> em 30 jan. 2012.

ITABORAHY, C. A Ritalina no Brasil: uma década de produção, divulgação e consumo. Dissertação (Mestrado em Medicina Social) - Instituto de Medicina Social, Universidade do Estado do Rio de Janeiro, Rio de Janeiro, 2009.

JENKINS, J.H. (Org.). Pharmaceutical Self: the global shaping of experience in an age of psychopharmacology. Santa Fe: School of Advanced Reseach, 2010.

LIMA, R. Somos Todos Desatentos? O TDA/H e a construção de bioidentidades. Rio de Janeiro: Relume-Dumará, 2005.

MATTOS, P. et al. Apresentação de uma versão em português para uso no Brasil do instrumento MTA-SNAP-IV de avaliação de sintomas de transtorno do déficit de atenção/ hiperatividade e sintomas de transtorno desafiador e de oposição. Rev. psiquiatr. Rio Gd. Sul [online], v.28, n.3, p. 290-297, 2006. Disponível em: <http://www.scielo.br/scielo. php?script $=$ sci_arttext $\&$ pid $=$ S0101-81082006000300008\&lng $=$ en $\&$ nrm $=$ iso $>$. Acesso em: 26 jul 2013. 
NATIONAL INSTITUTES OF HEALTH. Diagnosis and treatment of attention deficit hyperactivity disorder. NHI Consens Statement. V.16, n.2, p.1-37, 1998.

NOVARTIS. Ritalina (bula) Disponível em: <http://www4.anvisa.gov.br/base/visadoc/ BM/BM\%5B26162-1-0\%5D.PDF> Acesso em: ago 2012.

ORGANIZAÇÃO DAS NAÇÕES UNIDAS. Report of the International Narcotics Control Board for 2011. Statistics for 2010. Disponível em: <http://www.incb.org/pdf/annual report/2011/English/AR_2011_English.pdf> Acesso em: 29 mar 2012.

PARENS, E.; JOHNSTON, J. Facts, values, and attention-deficit hyperactivity disorder (ADHD): an update on the controversies. Child and Adolescent Psychiatry and Mental Health, v.3, n.1, p.1-17, 2009.

PASTURA, G.; MATTOS, P.; ARAUJO, A.P.Q.C. Prevalência do transtorno do déficit de atenção e hiperatividade e suas comorbidades em uma amostra de escolares. Arq. NeuroPsiquiatr. São Paulo, v.65, n.4a, dez. 2007. Disponível em <http://www.scielo.br/scielo. php?script $=$ sci_arttext $\&$ pid $=$ S0004-282X2007000600033\&lng=pt\&nrm $=$ iso $>$. Acesso em: 16 ago. 2013.

PETRYNA, A.; LAKOFF, A.; KLEINMAN, A. (Eds.). Global Pharmaceuticals: ethics, markets, practices. Durham, NC: Duke University Press, 2006.

RAFALOVICH, A. Framing the ADHD child: history, discourse and everyday experience. Tese (Doutorado em Sociologia) - The Faculty of Graduate Studies, Department of Anthopology \& Sociology, University of British Columbia, Vancouver, Canadá, 2002.

ROHDE, L.A.; BARBOSA, G.; TRAMONTINA, S.; POLANXZYK, G. Transtorno de déficit de atenção/hiperatividade. Revista Brasileira de Psiquiatria. v. 22, (Suplemento), p.7-11, 2000.

ROHDE, L.A.; HALPERN, R. Transtorno do déficit de atenção/hiperatividade: atualização. Jornal de Pediatria [Online] n.80, supl.2, p.S61-S70, 2004. Disponível em: <http://www.scielo.br/pdf/jped/v80n2s0/v80n2Sa08.pdf>. Acesso em: 12 mar. 2012.

ROSE, N. Disorders without borders? The expanding scope of psychiatric practice, BioSocieties: an interdisciplinary journal for social studies of life sciences, v.1, n.4, p.465-484, 2006.

ROSEMBERG, C.E. The tyranny of diagnosis: specific entities and individual experience. The Milbank Quarterly, v.80, n.2, p.237-260, 2002.

ROHDE, L.A.; HALPERN, R. Transtorno do déficit de atenção/hiperatividade: atualização. Jornal de Pediatria [Online] n.80, supl.2, p.S61-S70, 2004. Disponível em: <http://www.scielo.br/pdf/jped/v80n2s0/v80n2Sa08.pdf>. Acesso em: 12 mar. 2012.

ROHDE, L.A. et al. Transtorno de déficit de atenção/hipatividade. Revista Brasileira de Psiquiatria. v. 22, supl. 2, p.7-11, 2000. 
SANTOS (Cidade). Guia de Padronização de medicamentos. Santos, 2009. Disponível em: <http://www.santos.sp.gov.br/frame.php?pag=/saude/saude.php>. Acesso em: 20 dez. 2010.

SECRETÁRIO é preso por não fornecer remédio. G1 Notícias, 15 mai. 2007 Disponível em: <http://g1.globo.com/Noticias/Brasil/0,,MUL36648-5598,00.html>. Acesso em: 20 dez. 2011.

SINGH, I. A framework for understand trends in ADHD diagnosis and stimulant drug treatment: Schools and schooling as a case study. BioSocieties, v.1, p.439-452, 2006.

Not just naughty: 50 years of stimulant drug advertising. In: TONE, A.; WATKINS, E. (Orgs.). Medicating modern America: prescription drugs in history. New York: New York University Press, 2007. p.131-55.

\section{Nota}

${ }^{1}$ N. Domitrovic foi responsável pela coleta de dados na Farmácia Cidadã Metropolitana e colaborou na realização de entrevistas, na análise de dados, na elaboração e redação do artigo. L.V. Caliman é a coordenadora do projeto de pesquisa, realizou entrevistas e participou da análise de dados, elaboração e redação do artigo, fazendo também sua revisão final. 
An analysis of public dispensing of methylphenidate in Brazil: the case of Espirito Santo state

The Attention Deficit Hyperactivity Disorder (ADHD) expansion and the growth of the consumption of methylphenidate, commonly indicated for its treatment, are current challenges the public health sector in many parts of the world. This research aimed to investigate the public dispensing of methylphenidate hydrochloride by the Brazilian Unified Health System (SUS), stressing the case of the state of Espirito Santo (ES). A map of pharmaceutical care Policies in the Brazilian Federal Units was made based on telephone calls and consultations at their official websites. It was found that among all state's Pharmaceutical Care Management of the country only 4 , including the state of ES, have standardized lists for medicine dispensing in wich methylphenidate is included. The characteristics and variations in demand and consumption of methylphenidate registered in public pharmacies of ES between 2009 and 2011 were analyzed, in conjunction with information gathered in three semi-structured interviews with professionals in the Pharmaceutical Care Management. A significant increase in the consumption of this medicine via SUS was found throughout the analyzed period, with an asymmetric distribution between the eight public pharmacies of ES. These data highlight the need for careful analysis, focusing on the multiple aspects that interfere both in the formation of the diagnosis and in the demand for treatment, mainly in relation to the monitoring of public dispensation of methylphenidate. The study is essential in the public health context in order to support the policies formulation and the functioning of public services for ADHD.

> Key words: attention deficit disorder with hyperactivity; methylphenidate; pharmaceutical care. 